\title{
Combining Anatomical and Functional Networks for Neuropathology Identification: A Case Study on Autism Spectrum Disorder
}

\author{
Sarah Itani ${ }^{\mathrm{a}, \mathrm{b}, *}$, Dorina Thanou ${ }^{\mathrm{c}}$ \\ ${ }^{a}$ Fund for Scientific Research - FNRS (F.R.S.-FNRS), Brussels, Belgium \\ ${ }^{b}$ Department of Mathematics and Operations Research, Faculty of Engineering, \\ University of Mons, Mons, Belgium \\ ${ }^{c}$ Swiss Data Science Center, EPFL and ETH Zürich, Switzerland
}

\begin{abstract}
While the prevalence of Autism Spectrum Disorder (ASD) is increasing, research continues in an effort to identify common etiological and pathophysiological bases. In this regard, modern machine learning and network science pave the way for a better understanding of the neuropathology and the development of diagnosis aid systems. The present work addresses the classification of neurotypical and ASD subjects by combining knowledge about both the structure and the functional activity of the brain. In particular, we model the brain structure as a graph, and the resting-state functional MRI (rs-fMRI) signals as values that live on the nodes of that graph. We then borrow tools from the emerging field of Graph Signal Processing (GSP) to build features related to the frequency content of these signals. In order to make these features highly discriminative, we apply an extension of the Fukunaga-Koontz transform. Finally, we use these new markers to train a decision tree, an interpretable classification scheme, which results in a final diagnosis aid model. Interestingly, the resulting decision tree outperforms state-of-the-art methods on the publicly available Autism Brain Imaging Data Exchange (ABIDE) collection. Moreover, the anal-
\end{abstract}

\footnotetext{
* Corresponding author. University of Mons, Department of Mathematics and Operations Research, Rue de Houdain, 9, 7000 Mons, Belgium.

Email addresses: sarah.itani@umons.ac.be (Sarah Itani), dorina.thanou@epfl.ch (Dorina Thanou)
} 
ysis of the predictive markers reveals the influence of the frontal and temporal lobes in the diagnosis of the disorder, which is in line with previous findings in the literature of neuroscience. Our results indicate that exploiting jointly structural and functional information of the brain can reveal important information about the complexity of the neuropathology.

Keywords: graph signal processing; fMRI; autism spectrum disorder; explainable artificial intelligence.

\section{Introduction}

Understanding the human brain in all its complexity has always been a great challenge. Despite the undeniable progress made in the domain, some neuropathologies, such as Autism Spectrum Disorder (ASD), are characterized by the absence of a commonly defined etiology (Maximo et al., 2014). People with ASD manifest recurring behavioral patterns; they present impairments in language and communication which impede their social interactions (Autism Speaks, 2020). With the growing prevalence of ASD, especially in children, it becomes urgent to determine the neurophysiological bases of the disorder, and use this knowledge for an early and objective diagnosis. Towards that direction, data-driven techniques are expected to shed some light on explainable and interpretable markers that capture the complexity of the neuropathology and reveal interconnected patterns on brain activation that are related to the causes of the disorder (Vargason et al., 2020; Kassraian-Fard et al., 2016).

While there is no consensus on the pathophysiology of ASD, it is commonly accepted by the research community that the disorder can be partially explained by studying the brain network complex structure (Uddin et al., 2013). This structure is usually studied from a functional or a structural connectivity perspective. Functional Connectivity (FC) is typically derived from the correlation of signals such as the Blood-Oxygen-Level-Depedent (BOLD) ones, measured by resting-state functional Magnetic Resonance Imaging (rs-fMRI). Structural Connectivity (SC) is related to the detection of white matter pathways, tracked, 
for example, by Diffusion Tensor Imaging (DTI). Both FC and SC can be analyzed in terms of network to reveal organizing principles of the brain that are prevalent in ASD patients (Kazeminejad \& Sotero, 2019; Tolan \& Isik, 2018; Abraham et al., 2017; Chen et al., 2016; Rane et al., 2015; Kana et al., 2014; Maximo et al., 2014; Uddin et al., 2013; Vissers et al., 2012).

Given the complex nature of the brain and the paramount importance of both functional and structural connectivity, in this work, we combine both sources of information by borrowing tools from the emerging field of Graph Signal Processing (GSP) (Ortega et al., 2018; Shuman et al., 2013). GSP addresses the challenging problem of analyzing data living on an irregular domain, that can be naturally represented by a graph. The data lying on the nodes of the graph are considered as signals (e.g., fMRI time-series) that have a strong dependency on the graph topology (e.g., brain structural connectivity). Indeed, GSP allows to integrate both structural and functional brain data by studying the interplay between graphs and signals on graphs.

In particular, our goal is to classify ASD from neurotypical (NT) subjects. The graph in our application consists of a set of brain regions of interest, i.e., graph nodes, that are connected based on the anatomical distance distance between them. This graph structure remains constant over time. In contrast, the data observed on top of the graph (i.e., graph signals) are of time-varying nature, since they are generated by the BOLD fluctuations. The problem thus boils down to the classification of time-varying graph signals. In order to achieve that, we extend the GSP tools further, by adapting them to the particular settings. First, we study the frequency behavior of each BOLD signal in time by computing its Graph Fourier Transform (GFT). This step generates a set of time-varying GFT coefficients. These coefficients are then merged into a single connectivity matrix from which we extract discriminative graph frequency patterns, by using an extension of the Fukunaga-Koontz Transform (FKT). To eventually separate ASD from NT subjects, we use these features to train a decision tree, which results in a final diagnosis aid model. Experimental results on the publicly available ABIDE collection indicate that the proposed approach 
outperforms state-of-the-art methods, and confirm that exploiting jointly structural and functional information can indeed bring significant gain both in terms of performance and better understanding of the disorder.

\subsection{Related work}

Structural and functional information of the brain in ASD have already been extensively studied in the literature. For the sake of completeness, we mention here some representative works in the area. Chen et al. (2016) investigate resting-state FC in ASD over two frequency bands, namely the SLOw-4 (0.01-0.027 Hz) and SlOw-5 $(0.027-0.073 \mathrm{~Hz})$ bands. The FC values are used to train a Support Vector Machine (SVM) classifier. Interestingly, most of the discriminative features are located in the SLOW-4 band. Moreover, the analysis of the classification weights shows that the connections of the thalamus are the most discriminative. The work of Goch et al. (2014) uses structural network features such as the clustering coefficient and the betweeness centrality to achieve the classification of typically developing and ASD subjects through SVM. The achieved accuracy suggests the relevance of these structural features to understand the pathology. These findings are confirmed by Tolan \& Isik (2018), who compute graph measures on both fMRI and DTI-based networks at both global (e.g. diameter, modularity) and node levels (e.g., eccentricity, path length). The features are then used to achieve successful predictions through ensemble classification models consisting of SVM, decision tree, and $K$-nearest neighbor classifiers. Finally, a few studies have considered the joint analysis of both FC and SC, in quest of common functional and structural patterns of (hypo- or hyper-) connectivity in given brain areas (Ray et al., 2014; Mueller et al., 2013; Nair et al., 2013; Uddin et al., 2013). Such an example is the study of Mueller et al. (2013), which reveals common functional and structural impairments in the right temporo-parietal junction area, the left frontal lobe, and the bilateral superior temporal gyrus.

More recently, tools from the GSP framework have been proposed to combine structural and functional information in many brain related applications. Most 
of them focus on the analysis and classification of BOLD signals extracted from fMRI data, through the popular GFT. However, using GFT coefficients in their simple form is clearly not enough to capture activation patterns of the brain. For that reason, some studies perform the decomposition of signals into three components (i.e., low, medium and high frequency) in order to find patterns which are statistically significant to characterize cognitive flexibility (Medaglia et al., 2018) and motor skill (Huang et al., 2016). Along the same lines, Wang et al. (2018) suggest that GFT coefficients are not sufficient classification patterns, though used as inputs of a high-performing algorithm such as SVM. The work of Ktena et al. (2018) shows that GFT coefficients have better discriminating power when they are embedded within more complex patterns, learned through deep learning architectures such as convolutional neural networks. These findings suggest that the discriminative information brought by the time-varying GFT coefficients might be hidden in complex patterns. Thus, the challenge remains to discover these complex patterns, and at the same time, ensure a certain level of interpretability, i.e., the obtained patterns should be understood to a certain extent by humans, and more particularly clinicians in the context of medical data (Itani et al., 2019b; Doshi-Velez \& Kim, 2017).

\subsection{Contributions}

The contributions of our study are summarized as follows.

(1) We extend the GSP framework by proposing a way of discriminating timeseries in the graph Fourier domain. In that respect, we show that ASD can be predicted based on frequency patterns on the structural graph that are sophisticated, while remaining interpretable. Our results suggest that the differences between the ASD and NT subjects cannot be clearly attributed to specific graph frequency bands (i.e., typically low, middle and high bands), contrarily to what was previously put forward (Medaglia et al., 2018; Ménoret et al., 2017; Huang et al., 2016).

(2) Our discriminative patterns can be classified by a decision tree without the 


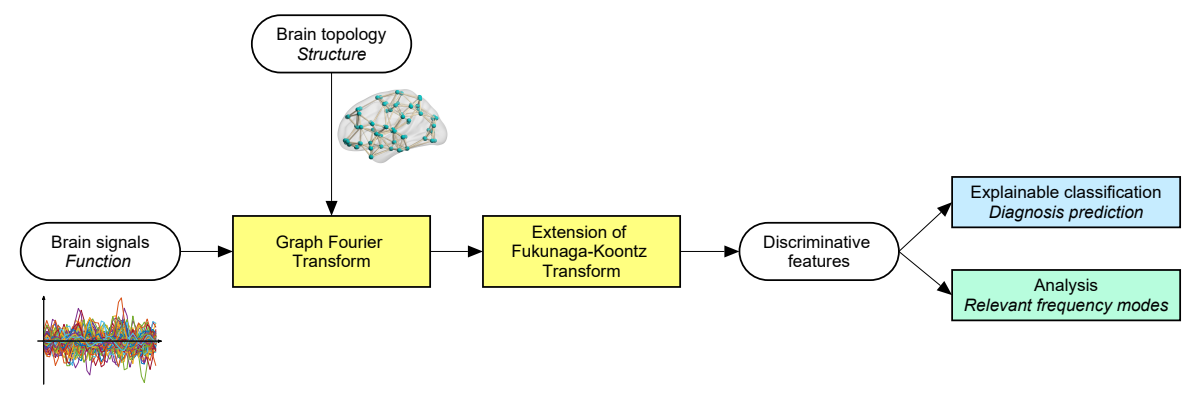

Figure 1: Overview of the proposed approach.

necessity of using more complex classification schemes, e.g., through deep learning as was considered by Ktena et al. (2018). This addresses to some extent the challenge of interpretability which arises for the development of diagnosis aid models (Itani et al., 2019b; Doshi-Velez \& Kim, 2017).

(3) In terms of classification accuracy, our framework outperforms other stateof-the-art methods that are based on either the structural or the functional connectivity, i.e., the Graph Fourier Transform - GFT (Ortega et al., 2018) and the Spatial Filtering Method - SFM (Subbaraju et al., 2017). Moreover, the interpretation of the results confirms previous findings of the neuroscience literature about ASD.

The remainder of the paper is structured as follows. First, we present the materials and methods for our study in Sec. 2. Then, we describe our experimental protocol in Sec. 3. We expose our results in Sec. 4, which are followed by a discussion in Sec. 5. Finally, we conclude the paper in Sec. 6 .

\section{Materials and methods}

In this section, we present our novel graph-based framework for the classification of the BOLD time-series (see Fig. 1), by describing in detail the tools and the building blocks. For the sake of simplicity, first, we present the notation that is used in the remainder of the paper (Subsec. 2.1). Then, in Subsec. 2.2, we introduce a methodology for jointly modeling the time-varying BOLD data 
and the underlying brain structure, by extending existing tools from GSP to our settings. Furthermore, in order to discriminate the BOLD time-series, in Subsec. 2.3, we extend the Fukunaga-Koontz transform to the graph-based representation of the previous subsection. Finally, we present the methodology used for classification and analysis (Subsec. 2.4).

\subsection{Settings and notations}

The classification problem that we tackle in this work consists of two classes: (i) NT, and (ii) ASD subjects. We denote by:

- $n_{T}$, the total number of subjects;

- $n_{A}$, the total number of ASD subjects;

- $n_{N}$, the total number of NT subjects.

Blood-Oxygen-Level-Dependent (BOLD) signals are available for each subject; they are provided for a set of $r$ Regions Of Interest (ROIs). For each subject $i$ $\left(i=\left\{1, \ldots n_{T}\right\}\right)$, we denote by:

- $T_{i}$, the number of time-points in the BOLD time-series;

- $\mathbf{X}_{i}$, the $r \times T_{i}$ matrix of BOLD time-series.

\subsection{Graph-based representation of BOLD time-series}

We model the structure of the brain as an undirected, weighted graph $\mathcal{G}$, where the set of nodes $\nu$ correspond to the brain regions of interest. The edges of the graph are defined by connecting close ROIs in terms of their topological distance in the brain. In particular, we define the weight $A_{u v}$ between two nodes $u, v$ of a brain graph $\mathcal{G}$ as the inverse of the distance $d_{u v}$ between the two nodes (i.e., regions of interest). Thus, the adjacency matrix $\mathbf{A}$ is such that:

$$
A_{u v}=d_{u v}^{-1} \quad \text { and } \quad A_{u u}=0 \text { for } \quad u, v=1, \ldots r .
$$




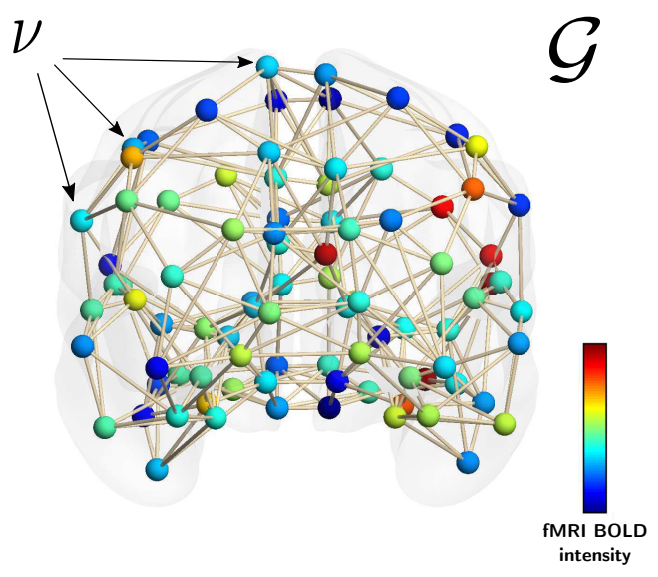

Figure 2: Representation of a graph signal, at a given time point. ${ }^{1}$

For each node, we keep only its $K$ nearest neighbors, while ensuring that the final graph is symmetric. The final adjacency matrix is computed as:

$$
\mathbf{A}^{\prime}=\frac{\mathbf{A}+\mathbf{A}^{T}}{2}
$$

For the sake of simplicity, in what follows, we denote this matrix as A. Note that the nearest-neighbor strategy is a good proxy for representing the brain topology (Alexander-Bloch et al., 2012; Bullmore \& Sporns, 2009). The combinatorial Laplacian operator (Shuman et al., 2013) is defined as $\mathbf{L}=\mathbf{D}-\mathbf{A}$ where $\mathbf{A}$ is the graph adjacency matrix, and $\mathbf{D}$ is a diagonal matrix containing the degree of each node, i.e., $\mathbf{D}_{k k}=\sum_{j} \mathbf{A}_{k j}$. .

Let us consider the activity of the brain at a given time instance. This involves observing the BOLD time-series issued by the ROIs at a given time point, i.e., a set of $r$ values contained in one of the column vectors of matrix $\mathbf{X}_{i}$ (see Subsec. 2.1). This set of values lying on the brain graph is usually denoted as a graph signal. Fig. 2 illustrates a 5-nearest neighbor topology of the brain, on top of which a graph signal is observed as an instantaneous measure of the brain activity.

\footnotetext{
${ }^{1}$ Note that the brain figures presented in the present paper were drawn with the BRAINNET SOFTwARE (Xia et al., 2013).
} 


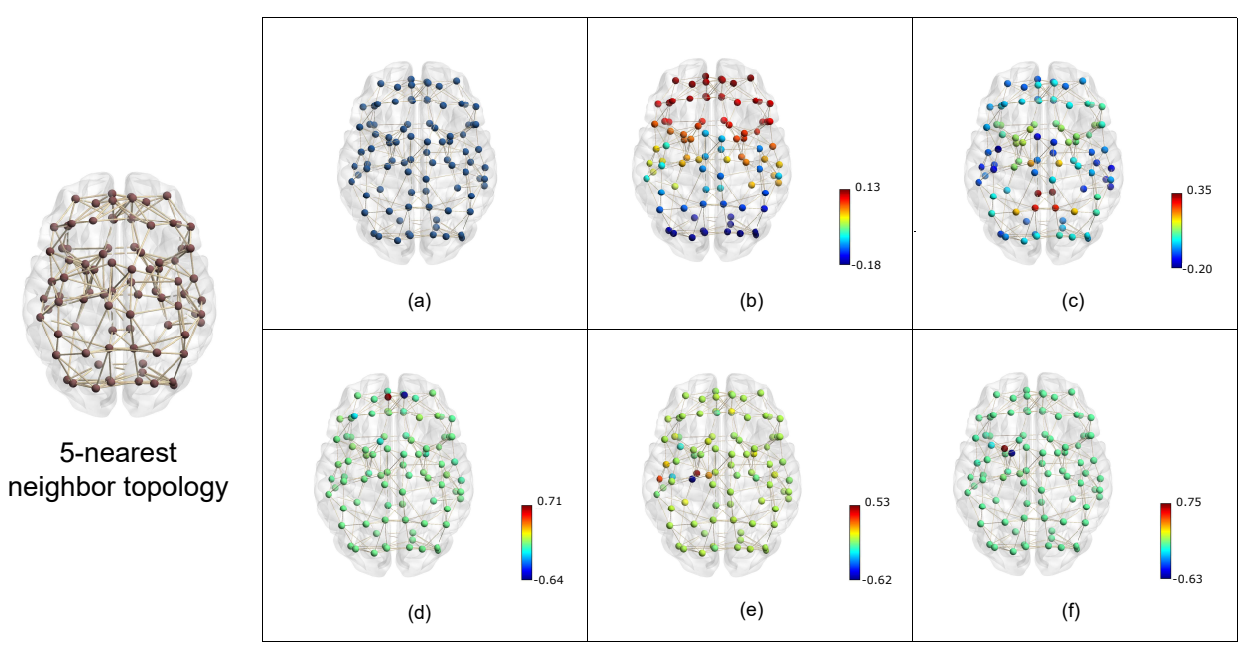

Figure 3: GFT basis illustrated on a 5-nearest neighbor topology consisting of 90 nodes (Tzourio-Mazoyer et al., 2002). The GFT modes (or frequencies) presented from (a) to (f) correspond to modes 1, 2, 10, 70, 75 and 90 respectively. The color represents the intensity of the signal (eigenvector of the Laplacian) on the graph. The highest the GF mode, the more fluctuating the signal values on neighboring nodes.

The spectral domain representation can reveal significant information about the characteristics of graph signals. In particular, the Graph Fourier Transform (GFT) provides a frequency analysis of these signals, that is based on the graph Laplacian operator. The matrix of eigenvectors $\mathbf{V}$ of the Laplacian operator can be used to perform a harmonic analysis of signals that live on the graph (i.e., graph signals), and the corresponding eigenvalues carry a notion of frequency (Shuman et al., 2013). Indeed, the eigenvectors of the Laplacian consist of the Fourier basis that can be used for analyzing graph signals. Fig. 3 shows the GFT modes of a 5 -nearest neighbor brain graph. From $(a)$ to $(f)$, we present different graph frequency modes, in ascending order of eigenvalues, i.e., graph frequencies. The eigenvectors associated with low eigenvalues (i.e., low frequency) are smooth on the graph, which implies that they are changing slowly across nodes that are connected by an edge. Mode $(a)$ corresponds to a constant graph signal. 
In the present work, we model our time-varying graph signals through their GFT coefficients. In particular, for each subject $i$, we compute

$$
\hat{\mathbf{X}}_{i}=\mathbf{V}^{\mathbf{T}} \mathbf{X}_{i}
$$

Each column of $\hat{\mathbf{X}}_{i}$ relates to the GFT coefficients computed at each time point. Projecting the signals in the graph Fourier domain can be considered as a way of decorrelating the signals in that basis. In order to understand the variation of each GFT coefficient over time, we normalize the GFT coefficients at a specific instance of time, in such a way that the relative importance of each frequency component is revealed. More specifically, we normalize the columns of $\hat{\mathbf{X}}_{i}$ by subtracting the mean of each column and dividing by its energy i.e., the $L 2$ norm (see Appendix A.1, Eq. A.1). The resulting matrices are denoted by $\mathbf{Y}_{i}$. We then merge the normalized GFT coefficients over time in order to reveal some frequency patterns of the time-series in the graph Fourier domain. We achieve that by computing an approximation of the sample covariance matrix, that is given by:

$$
\mathbf{S}_{i}=\frac{\mathbf{Y}_{i} \mathbf{Y}_{i}^{\mathbf{T}}}{\operatorname{tr}\left(\mathbf{Y}_{i} \mathbf{Y}_{i}^{\mathbf{T}}\right)}
$$

The mean joint expectancy matrix over the patients is computed as:

$$
\overline{\mathbf{S}}=\frac{1}{n_{T}} \sum_{i=1}^{n_{T}} \frac{\mathbf{Y}_{i} \mathbf{Y}_{i}^{\mathbf{T}}}{\operatorname{tr}\left(\mathbf{Y}_{i} \mathbf{Y}_{i}^{\mathbf{T}}\right)}
$$

We compute similarly the mean joint expectancy matrices for the ASD and NT subjects, which are denoted respectively as $\overline{\mathbf{S}}^{\mathbf{A}}$ and $\overline{\mathbf{S}}^{\mathbf{N}}$. It follows that:

$$
\overline{\mathbf{S}}=\frac{n_{A}}{n_{T}} \overline{\mathbf{S}}^{\mathbf{A}}+\frac{n_{N}}{n_{T}} \overline{\mathbf{S}}^{\mathbf{N}}=\frac{n_{A}}{n_{T}} \overline{\mathbf{S}}^{\mathbf{A}}+\left(1-\frac{n_{A}}{n_{T}}\right) \overline{\mathbf{S}}^{\mathbf{N}} .
$$

For the remainder of the development, we denote by $\alpha_{A}$ and $\alpha_{N}$ respectively, the factors $\frac{n_{A}}{n_{T}}$ and $\frac{n_{N}}{n_{T}}$, and with $\alpha_{N}=1-\alpha_{A}$. Eq. 5 can be reformulated as:

$$
\overline{\mathbf{S}}=\alpha_{\mathrm{A}} \overline{\mathbf{S}}^{\mathbf{A}}+\alpha_{\mathrm{N}} \overline{\mathbf{S}}^{\mathbf{N}}
$$

The mean joint expectancy matrix is thus expressed as a positive linear combination of both mean joint expectancy matrices of ASD and NT subjects. 


\subsection{Finding a discriminative subspace}

After computing a representative matrix that captures the temporal evolution of the graph spectral components of the BOLD fluctuations for each category, we need to classify the subjects in one of the two categories. To achieve this, we propose an extension of the Fukunaga-Koontz Transform (FKT) (Fukunaga, 2013; Fukunaga \& Koontz, 1970) to time-varying graph signals. This method achieves separation of two classes by relying on the simultaneous diagonalization of the covariance matrices (Huo, 2004). Such a diagonalization results in a set of common eigenvectors, along which the classes have complementary eigenvalues. This means that a significant dimension for a class is less relevant for the other, and vice-versa. Reduction is performed in keeping the dimensions associated with the highest eigenvalues for each class. Our proposed extension of the method to our graph settings consists of the following three steps.

- Whitening. First, we need to decorrelate the data by means of a whitening operator. Let us consider the eigen-decomposition of the matrix $\overline{\mathbf{S}}$ :

$$
\mathrm{Q}^{\mathrm{T}} \overline{\mathbf{S}} \mathbf{Q}=\Lambda \quad \Leftrightarrow \quad \overline{\mathrm{S}}=\mathrm{Q} \Lambda \mathrm{Q}^{\mathrm{T}}
$$

As $\overline{\mathbf{S}}$ is symmetric, it holds that $\mathbf{Q}^{-\mathbf{1}}=\mathbf{Q}^{\mathbf{T}}$. It can be shown that $\overline{\mathbf{S}}$ has a zero eigenvalue due to the column-wise normalization of the GFT coefficients (see Appendix A.2). We consider, without loss of generality, that the eigenvalues of $\overline{\mathbf{S}}$ are sorted in ascend order. The diagonal matrix $\boldsymbol{\Gamma}$ is defined as:

$$
\Gamma_{11}=1 \text { and } \Gamma_{i i}=\Lambda_{i i}^{-1 / 2} \text {, with } i=2, \ldots r
$$

Thus, if we set $\mathbf{Q}_{\mathbf{2}}=\boldsymbol{\Gamma}^{\mathbf{T}} \mathbf{Q}^{\mathbf{T}}$, we have $\mathbf{Q}_{\mathbf{2}} \overline{\mathbf{S}} \mathbf{Q}_{\mathbf{2}}{ }^{\mathbf{T}}=\operatorname{diagonal}\left[\mathbf{0}, \mathbf{I}_{\mathbf{r}-\mathbf{1}}\right]$. The matrix diagonal $\left[\mathbf{0}, \mathbf{I}_{\mathbf{r}-\mathbf{1}}\right]$ has a first diagonal element equal to 0 and the remaining ones equal to one. Using the above developments, Eq. 6 is rewritten as:

$$
\begin{aligned}
& \mathbf{Q}_{\mathbf{2}} \overline{\mathbf{S}} \mathbf{Q}_{\mathbf{2}}^{\mathbf{T}}=\alpha_{\mathrm{A}} \mathbf{Q}_{\mathbf{2}} \overline{\mathbf{S}} \mathbf{Q}_{\mathbf{2}}^{\mathbf{T}}+\alpha_{\mathrm{N}} \mathbf{Q}_{\mathbf{2}} \overline{\mathbf{S}}^{\mathbf{N}} \mathbf{Q}_{\mathbf{2}}^{\mathbf{T}} \\
& \Leftrightarrow \operatorname{diagonal}\left[\mathbf{0}, \mathbf{I}_{\mathbf{r}-\mathbf{1}}\right]=\alpha_{\mathrm{A}} \overline{\mathbf{S}}^{\mathbf{A}^{\prime}}+\alpha_{\mathrm{N}} \overline{\mathbf{S}}^{\mathbf{N}^{\prime}} .
\end{aligned}
$$


. Simultaneous diagonalization of the whitened matrices. Then, we have to find a transform which keeps the overall effect of whitening, while making the variance of the classes emerge in a complementary way. This operation corresponds to the simultaneous diagonalization of the joint expectancy matrices $\left(\overline{\mathbf{S}}, \overline{\mathbf{S}}^{\mathbf{A}}, \overline{\mathbf{S}}^{\mathbf{N}}\right)$ which are actually related by Eq. 6 . The diagonalization is based on the results of the Newcomb's theorem (see Appendix A.3), and performed through a matrix $\mathbf{T}_{\mathbf{2}}$ such that Eq. 7 can be reformulated as:

$$
\begin{gathered}
\mathbf{T}_{\mathbf{2}}^{\mathbf{T}} \text { diagonal }\left[\mathbf{0}, \mathbf{I}_{\mathbf{r}-\mathbf{1}}\right] \mathbf{T}_{\mathbf{2}}=\alpha_{\mathrm{A}} \mathbf{T}_{\mathbf{2}}^{\mathbf{T}} \overline{\mathbf{S}}^{\mathbf{A}^{\prime}} \mathbf{T}_{\mathbf{2}}+\alpha_{\mathrm{N}} \mathbf{T}_{\mathbf{2}}^{\mathbf{T}} \overline{\mathbf{S}}^{\mathbf{N}^{\prime}} \mathbf{T}_{\mathbf{2}} \\
\Leftrightarrow \operatorname{diagonal}\left[\mathbf{0}, \mathbf{I}_{\mathbf{r}-\mathbf{1}}\right]=\alpha_{\mathrm{A}} \overline{\mathbf{S}}^{\mathbf{A}^{\prime \prime}}+\alpha_{\mathrm{N}} \overline{\mathbf{S}}^{\mathbf{N}^{\prime \prime}} .
\end{gathered}
$$

The matrices $\overline{\mathbf{S}}^{\mathbf{A}^{\prime \prime}}$ and $\overline{\mathbf{S}}^{\mathbf{N}^{\prime \prime}}$ are diagonal. Their non-zero eigenvalues, multiplied respectively by $\alpha_{\mathrm{A}}$ and $\alpha_{\mathrm{N}}$, are complementary and sum to unity. The details for the construction of matrix $\mathbf{T}_{\mathbf{2}}$ are provided in Appendix A.4.

Computation of the projection matrix. All the above operations can be summarized through a final projection matrix $\mathbf{P}$ such that:

$$
\begin{aligned}
& \mathbf{P} \overline{\mathbf{S}} \mathbf{P}^{\mathbf{T}}=\alpha_{\mathrm{A}} \mathbf{P} \overline{\mathbf{S}}^{\mathbf{A}} \mathbf{P}^{\mathbf{T}}+\alpha_{\mathrm{N}} \mathbf{P} \overline{\mathbf{S}}^{\mathbf{N}} \mathbf{P}^{\mathbf{T}} \\
& \Leftrightarrow \operatorname{diagonal}\left[\mathbf{0}, \mathbf{I}_{\mathbf{r}-\mathbf{1}}\right]=\alpha_{\mathrm{A}} \overline{\mathbf{S}}^{\mathbf{A}^{\prime \prime}}+\alpha_{\mathrm{N}} \overline{\mathbf{S}}^{\mathbf{N}^{\prime \prime}}
\end{aligned}
$$

with

$$
\mathbf{P}=\mathbf{T}_{2}^{\mathrm{T}} \mathbf{Q}_{2}=\mathbf{T}_{2}^{\mathrm{T}} \Gamma^{\mathrm{T}} \mathbf{Q}^{\mathrm{T}}
$$

Thus, we end up with a matrix which can be used to project each patient's matrix of time-varying GFT coefficients in a space where the ASD and NT classes have complementary mean joint expectancy matrices. This explains why the subspace is discriminative: each class may be expressed through a subset of dimensions along which the variance of the related data is high.

We note that the above extension is inspired by the Spatial Filtering Method (SFM). The method is introduced by Subbaraju et al. (2017) as an extension of the FKT for the classification of ASD patients, based on their rs-fMRI data. In fact, SFM addresses a two-class classification problem by projecting the BOLD time-series of the instances in a space defined by the FKT such that they are 
distinctively separable. We believe that adding the structural information to the SFM framework, and studying patterns in the graph Fourier domain, can provide more insight on brain behavior in ASD patients.

\subsection{Performing classification and analysis}

The projection matrix $\mathbf{P}$ obtained from the previous subsection can be used to classify the GFT coefficients of the BOLD fluctuations. Thus, we project the normalized GFT coefficients $\mathbf{Y}_{i}$ (see Subsec. 2.2) into the discriminative matrix $\mathbf{P}$ (see Eq. 8):

$$
\mathbf{Z}_{i}=\mathbf{P} \cdot \mathbf{Y}_{i}
$$

Classification is then achieved by training a model on the variance of the projected GFT coefficients. Our classification scheme is based on the decision tree mainly because of the interpretability that it provides, which is crucial for diagnosis prediction. This quality is also enhanced by the interpretability of the features. Indeed, from Eq. 9, we notice that the rows of the discriminative matrix act as filters on the GFT coefficients contained in each column of matrix $\mathbf{Y}_{i}$. The result is a weighted sum of the GFT coefficients in the projection space on each of its dimensions. Hence, the analysis of $\mathbf{P}$ reveals the graph Fourier modes that contribute the most to the discriminative features, and thus to the diagnosis predictions.

Finally, we note that the variance of the elements included in the first row of $\mathbf{Z}_{i}$ does not carry any discriminative information. Indeed, along this first dimension, both classes are associated with a zero eigenvalue (see Eq. 8), due to the

singularity of the mean joint expectancy matrix (see Eq. 4). In other terms, circumventing this issue was achieved at the expense of a discriminative dimension in the projection space, through a transform which is not orthonormal.

\section{Experimental protocol}

Fig. 4 depicts our experimental protocol which consists of the data (Subsec. 3.1), the choice of a brain topology (Subsec. 3.2), the assessment modalities 
(Subsec. 3.3), and a tuning strategy for classification (Subsec. 3.4). These several aspects are introduced in the following.

\subsection{Data}

In this study, we consider the ABIDE I preprocessed dataset (Di Martino et al., 2014; Craddock et al., 2013). It includes BOLD time-series extracted from rs-fMRI through a preprocessing pipeline which is fully detailed on the web platforms related to the ABIDE collection (Preprocessed Connectomes Project, 2014; Autism Brain Imaging Data Exchange, 2013). The time-series considered in our work were preprocessed according to the C-PAC pipeline, which involves the following main steps: basic processing, noise signal removal, global regression, band-pass filtering $(0.01-0.1 \mathrm{~Hz})$, registration, time-series extraction (Preprocessed Connectomes Project, 2014). The selected mean time-series correspond to the Automated Anatomical Labeling atlas on 90 regions of interest (AAL90), i.e., $r=90$ (Tzourio-Mazoyer et al., 2002). The AAL90 parcellation is documented in Appendix A.5.

Though exhaustive, the ABIDE dataset presents a high degree of heterogeneity, mainly because of the conditions under which the fMRI acquisition was operated (i.e., eyes closed/opened) as well as the demographic distribution. Thus, to ensure consistent and reliable results, we consider patients who meet the following inclusion criteria:

- eyes opened during fMRI acquisition;

- less than 18 years old;

- less than $0.2 \mathrm{~mm}$ in mean framewise displacement.

The corresponding data subset consists of a total of 452 subjects, with respectively $251 \mathrm{NT}$ and $201 \mathrm{ASD}$ subjects.

\subsection{Brain topology definition}

As discussed in Sec. 2.2, we define the brain graph topology by following a nearest neighbor strategy. In particular, we focus on a two-nearest neighbor 


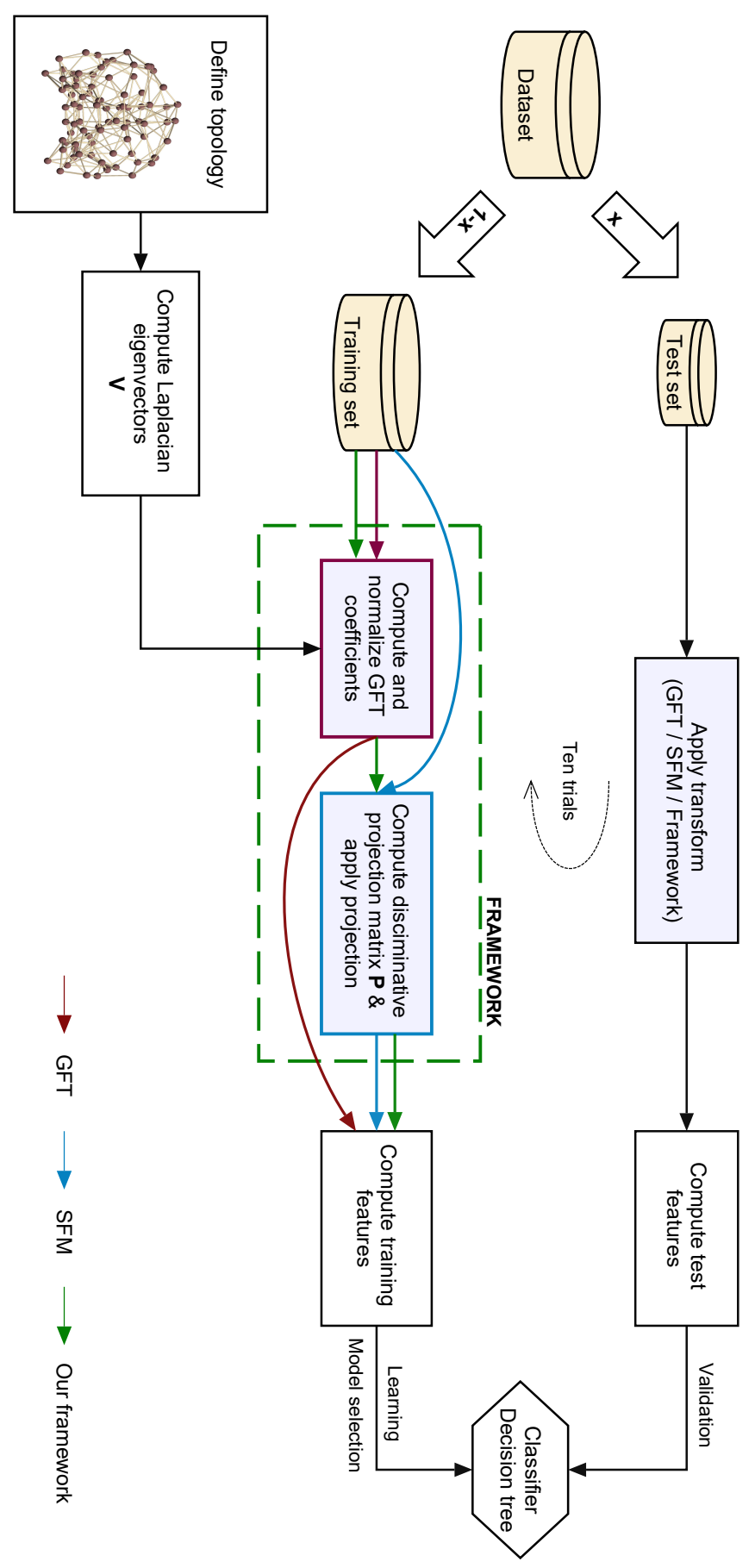

Figure 4: Experimental pipeline. 


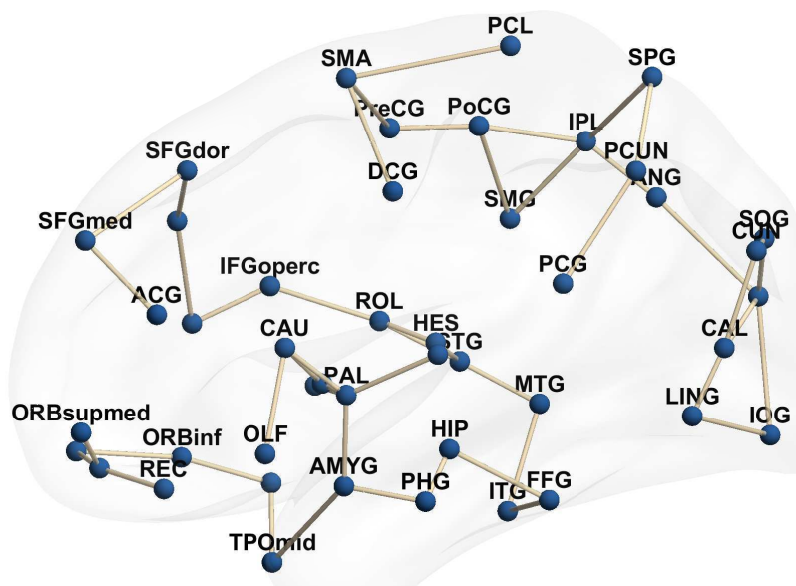

Figure 5: Two-nearest neighbor topology in the right hemisphere.

topology, which drew our attention in terms of spatial distribution of the ROIs. Indeed, this topology roughly divides the brain in two parts: the fronto-temporal areas on the one side, and the parieto-occipital areas on the other side (see Fig. 5). This choice of topology is meaningful from the neuroscience point of view. Fronto-temporal areas have been associated to dysfunctions and structural abnormalities in ASD subjects (Hirata et al., 2018; Lauvin et al., 2012; Poustka et al., 2012; Goldberg et al., 1999). Actually, the frontal lobe plays an important role in the regulation of our emotions, as it conditions our personality and our ability in making decisions (Abhang et al., 2016). As far as the temporal lobe is concerned, it is notably involved in processing language and emotion, through the amygdala (Abhang et al., 2016; Baars \& Gage, 2010).

\subsection{Assessment settings}

The initial dataset is split into training and test sets (see Fig. 4). This is achieved by picking randomly a fraction $x$ of the total number of instances to constitute a test set. The remaining part is left for training. We consider ten trials, and report the average test accuracy. Moreover, we compare our 
framework with two different approaches: the first is based on the classification of the GFT coefficients (see Sec. 2.2) and the second is based on SFM.

As shown in Fig. 4, within each trial, the training data are processed through GFT, SFM and our framework. For each patient, training features are derived from the projected BOLD time-series according to the following procedures.

- Concerning the GFT pipeline, we compute the variance of the normalized GFT coefficients over time. As there are 90 ROIs, there are 90 frequency modes, which result in 90 training features per time instance, per subject. We also consider the set of variances related to equally-defined frequency bands, i.e., low, middle and high frequency modes. This method has been used for successful understanding of cognitive flexibility (Medaglia et al., 2018) and motor skill (Huang et al., 2016).

- For both SFM and our framework, we consider the variance of the projected data (see Eq. 9) related to the $m$ most significant dimensions for each group, with $m \in[2,5]$. Thus, there is a total of $2 \times m$ training features.

\subsection{Classifier tuning}

In order to classify the subjects into NT and ASD we use a decision tree on the set of training features within each trial. In terms of implementation, we consider the C4.5 implementation of WEKA (Frank et al., 2016). All parameters are kept at their default values, except from the minimal number of instances per leaf, which is a parameter conditioning the decision tree depth. Tuning is

performed through an inner cross-validation launched on each training split to select the best corresponding parameter value.

\section{Results}

\subsection{Performance assessment and comparison with reference methods}

Fig. 6 shows the best averaged test accuracies achieved by each method (across all GSP modalities), according to the procedure described in Sec. 3.3, 


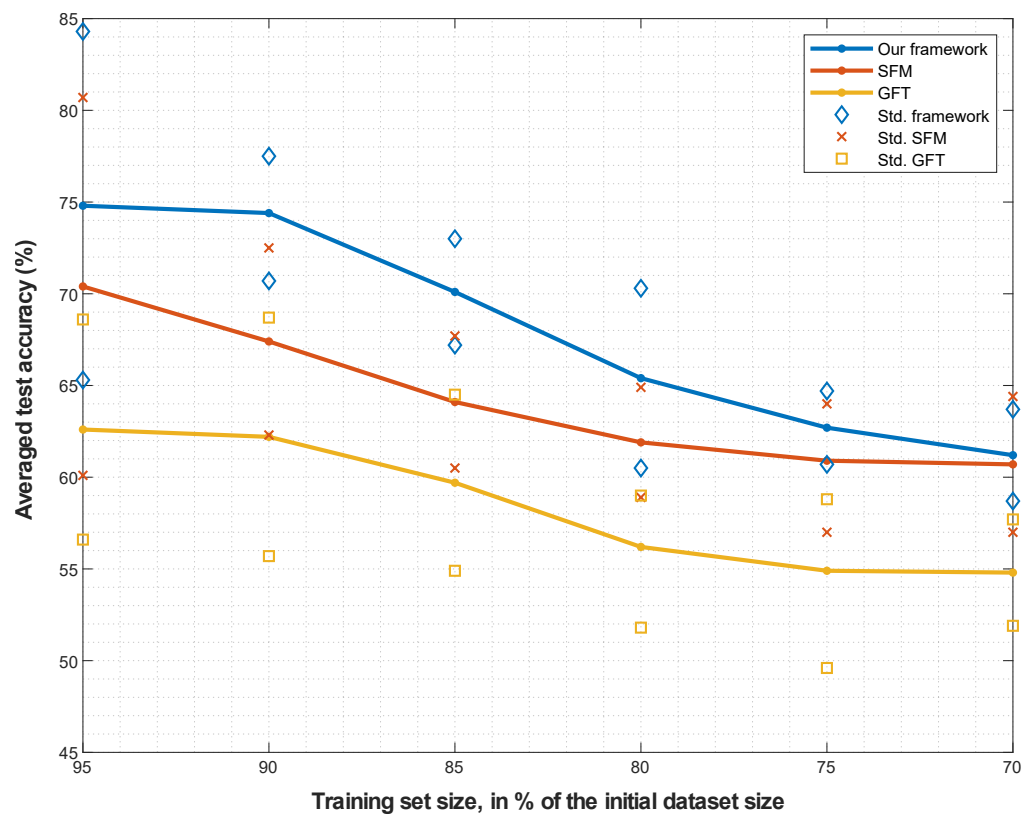

Figure 6: Performances of the methods for different training set sizes. The markers denote the standard deviation (Std.) intervals.

for different sizes of the training set (from $95 \%$ to $70 \%$, per step of $5 \%$ ). The markers around each curve delineate the standard deviation intervals related to the measures of accuracy.

As the training set size decreases, the performance deteriorates significantly in all the methods. Such a trend is expected, notably in the case of SFM and our framework, which are both based on the estimation of a covariance matrix through the sample covariance. Note that the standard deviation decreases as more data is available for testing.

We draw two main observations from Fig. 6 .

- With a performance gap of up to $12 \%$, our framework significantly outperforms the GFT-based approach, which suggests that the discriminative information is hidden in more complex patterns, revealed by combining the GFT coefficients.

- The performance gap between SFM and our framework is less pronounced, 


\begin{tabular}{c|ccc}
\hline$m$ & SFM (\%) & Ours (\%) & $p$-value \\
\hline 2 & $69.1 \pm 7.4$ & $\mathbf{7 3 . 5} \pm 6.9$ & 0.026 \\
3 & $70.4 \pm 10.3$ & $\mathbf{7 4 . 8} \pm 9.5$ & 0.016 \\
4 & $69.6 \pm 10.1$ & $\mathbf{7 3 . 0} \pm 6.4$ & $<0.001$ \\
5 & $67.4 \pm 9.2$ & $\mathbf{7 1 . 3} \pm 8.1$ & $<0.001$ \\
\hline
\end{tabular}

Table 1: Comparison between SFM and our framework in terms of averaged test accuracy.

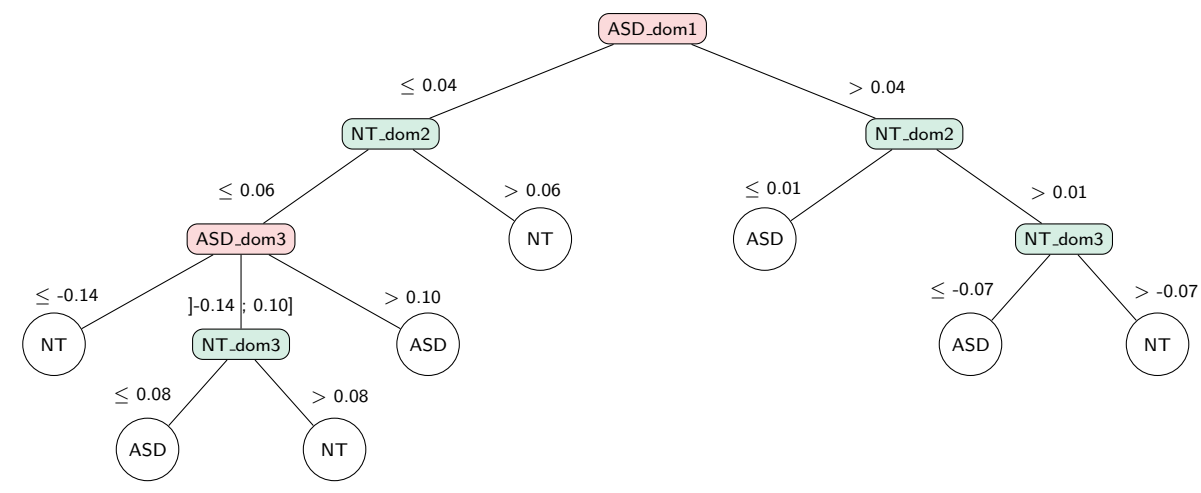

Figure 7: Decision tree based on the projected coefficients of our framework; the subdivisions are related to the log-variance values.

but almost always significant. While both methods implement the FKT principle, it seems that the topological information brought by our framework influences positively the results, especially in the high-data regime.

Table 1 compares our approach with SFM, and presents in detail the averaged test accuracies obtained by keeping $95 \%$ of the initial dataset for training, for different assessment modalities. We also include the $p$-values related to the Student's $t$-test. The hypothesis of equal performances is rejected for all the modalities, with $p$-values inferior to $5 \%$.

Finally, we study the interpretability of the features obtained by our framework. Fig. 7 presents the decision tree achieved on the basis of the three most discriminative dimensions $(m=3)$ for each of the two groups. By $A S D_{-} d o m$ 


\begin{tabular}{c|ccc||cc}
\hline$m$ & rand WFC & UC & WFC & SFM & 2-NN \\
\hline $\mathbf{2}$ & $67.4 \pm 9.4$ & $66.1 \pm 9.5$ & $67.8 \pm 9.0$ & $69.1 \pm 7.4$ & $73.5 \pm 6.9$ \\
$\mathbf{3}$ & $66.1 \pm 9.5$ & $69.1 \pm 6.3$ & $67.8 \pm 7.8$ & $70.4 \pm 10.3$ & $74.8 \pm 9.5$ \\
$\mathbf{4}$ & $64.8 \pm 7.9$ & $65.2 \pm 9.9$ & $70.0 \pm 10.4$ & $69.6 \pm 10.1$ & $73.0 \pm 6.4$ \\
$\mathbf{5}$ & $67.4 \pm 7.1$ & $63.9 \pm 8.3$ & $65.2 \pm 11.8$ & $67.4 \pm 9.2$ & $71.3 \pm 8.1$ \\
\hline
\end{tabular}

Table 2: Influence of the topology on the predictive performances

(respectively NT_dom) we denote a dimension along which the ASD group (resp. NT group) has a large variance; the value related to this feature refers to the discriminative power of the dimension. For instance, ASD_dom1 (resp. NT_dom1) refers to the first strongly dominant dimension for the ASD group (resp. NT group). The subdivisions are based on the variance (over time) of the coefficients along the dimension in question. More precisely, for a given patient, the decision tree checks the feature corresponding to $A S D_{-} d o m 1$. If the variance along this dimension is high, the algorithm examines the features corresponding to NT_dom2. A low variance along this dimension implies an ASD diagnosis.

\subsection{Influence of the topology}

In order to understand the influence of the topology on the classification performance, we perform additional experiments, by considering alternative connectivity matrices:

- a Weighted Fully Connected (WFC) topology which is generated by connecting all the nodes of the graph to each other, in assigning as a weight the inverse distance (see Subec. 2.2). We also consider a random WFC (rand WFC);

- a Uniformly Connected (UC) topology, generated by connecting all the nodes to each other, in assigning a constant and unit weight to all the connections.

Table 2 presents the corresponding predictive performances, which are statistically different from the results achieved with a 2 -NN topology $(p<0.05$, see 


\begin{tabular}{cccc}
\hline$m$ & rand WFC & UC & WFC \\
\hline $\mathbf{2}$ & 0.010 & 0.006 & 0.002 \\
$\mathbf{3}$ & 0.005 & 0.002 & 0.008 \\
$\mathbf{4}$ & 0.002 & 0.003 & 0.033 \\
$\mathbf{5}$ & 0.002 & 0.005 & 0.005 \\
\hline
\end{tabular}

Table 3: $p$-values related to the comparison with our 2-NN-based framework (Student's $t$-test)

Table 3). For completeness, we show the results of the SFM method. It appears that introducing a form of structural information leads to different results from those of the SFM method. As the accuracies reported in Table 2 suggest, the knowledge-guided choice of the 2-NN topology proves beneficial in this respect. On the contrary, the projection of the BOLD time-series over a random structure (i.e., rand WFC) or a uniformly connected structure (i.e., UC) reduces the classification performances. Finally, it is worth noting that the performances achieved with the WFC topology are inferior to those achieved in the case of 2NN. This shows that probably the edges added to the 2-NN graph, i.e., non-local interactions, add some noise in the process.

\subsection{Results for the adult population}

The population targeted by our case study relates to adolescents (less than 18 years old). For assessment purposes, we consider another subsample of the ABIDE dataset which includes adults. This subsample follows the same inclusion criteria as those presented in Sec. 3.1, except for the age (superior to 18 years old in this case). The sample includes a total of 130 subjects, with 63 ASD and 67 neurotypical subjects. Given the limited size of the dataset, we consider a Leave-One-Out-Cross-Validation (LOOCV) procedure, and report the results for several values of parameter $m$, including those acquired using the entire training features. The results are reported in Table 4 for SFM and our framework based on a 2-NN topology. The methods are statistically different for all the tested modalities, with $p$-values strictly inferior to 0.001 . 


\begin{tabular}{l|cc}
\hline$m$ & SFM (\%) & Ours (\%) \\
\hline 45 & 54.6 & $\mathbf{6 6 . 9}$ \\
12 & 55.4 & $\mathbf{6 7 . 7}$ \\
9 & 52.3 & $\mathbf{6 7 . 7}$ \\
6 & 52.3 & $\mathbf{5 3 . 8}$ \\
\hline
\end{tabular}

Table 4: LOOCV accuracies achieved by SFM and our framework over the adult population

Note that the performances achieved over the adult population are inferior to those reached on the adolescent population. This is certainly attributable to the reduced size of the adult dataset, which includes 130 subjects against 452 subjects in the adolescent sample. Yet the ASD population is quite heterogeneous in profiles given the extent of the spectrum. The appropriate representation of the neuropathology thus requires a large amount of data, especially as the adult population covers here a large range of ages (from 18 to 50 years old). Despite these unfavorable conditions, we note that our framework outperforms the SFM method, with a performance gap that reaches up to $15 \%$.

\section{Discussion}

In this section, we discuss different aspects of the proposed framework and the achieved results. First, we analyze the discriminative features, in order to reveal and interpret the corresponding brain patterns (Subsec. 5.1). Then, we compare our results with the state of the art for the ABIDE dataset (Subsec. 5.2). Finally, we discuss the limitations of the present study and propose future directions accordingly (Subsec. 5.3).

\subsection{Interpretation of the discriminative features}

The results obtained from the proposed framework confirm that considering the structure-function interplay is crucial in classifying ASD and NT subjects. This pertinent information is however hidden in discriminative patterns which are made of complex combinations of the graph Fourier modes. As explained 


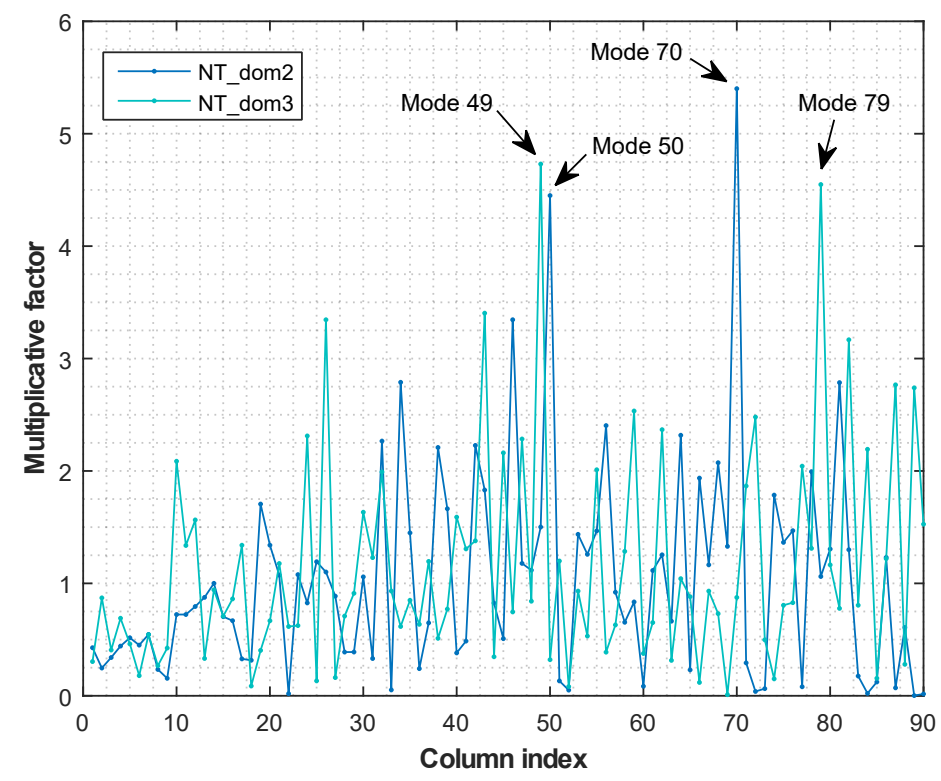

Figure 8: Matrix P: interpretation of the rows - NT-dominant dimensions.

in Sec. 2.4, the analysis of the projection matrix $\mathbf{P}$ allows us to understand how these combinations are made, and which modes contribute the most to the discriminative features. Figs. 8 and 9 show the values of the matrix $\mathbf{P}$ rows (in absolute values) which correspond to the dimensions considered by the decision tree of Fig. 7.

Fig. 8 is related to the set of weights applied on the GFT coefficients for their projection on the NT-dominant dimensions used by the predictive model (see Fig. 7). We notice that some weights are dominant over the others of the same raw, i.e., some GFT coefficients are, in terms of absolute values, more amplified than others in the final projection space. This allows to point out the corresponding graph Fourier modes, belonging to different levels of frequency (i.e., low, medium, high), as key patterns for classification, whose corresponding weights are deviating from the mean by more than 2.5 times the standard deviation. The same observation is valid in Fig. 9 for the ASD-dominant dimensions.

Given the above, we are lead to an interesting interpretation of the results. 


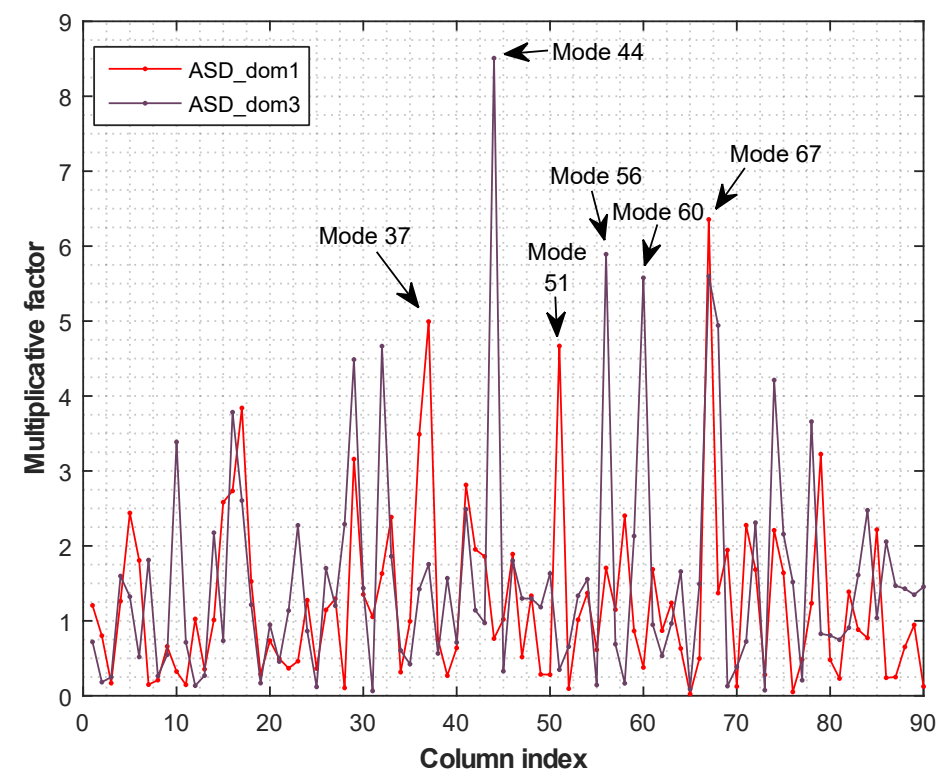

Figure 9: Matrix P: interpretation of the rows - ASD-dominant dimensions.

Indeed, by computing the variance of the projected GFT coefficients, we actually measure the variability over time of the presence of some graph Fourier modes in the fMRI signals. These modes may be seen as frequency signatures of the NT/ASD conditions. This constitutes another difference with the SFM method which allows to point out isolated prominent regions (Subbaraju et al., 2017).

The analysis of the graph Fourier modes pointed out in Fig. 8 reveals three out of the four signatures which correspond to a predominant activity in the parieto-occipital regions (see Fig. 10). Regarding the significant modes of the ASD population which are pointed out in Fig. 9, they are all related to high levels of activity in the fronto-temporal areas in ASD subject (see Fig. 11). This result is consistent with the previous findings reported in the literature of neuroscience about the influence of the frontal and temporal lobes in ASD (Hirata et al., 2018; Lauvin et al., 2012; Poustka et al., 2012; Goldberg et al., 1999). 

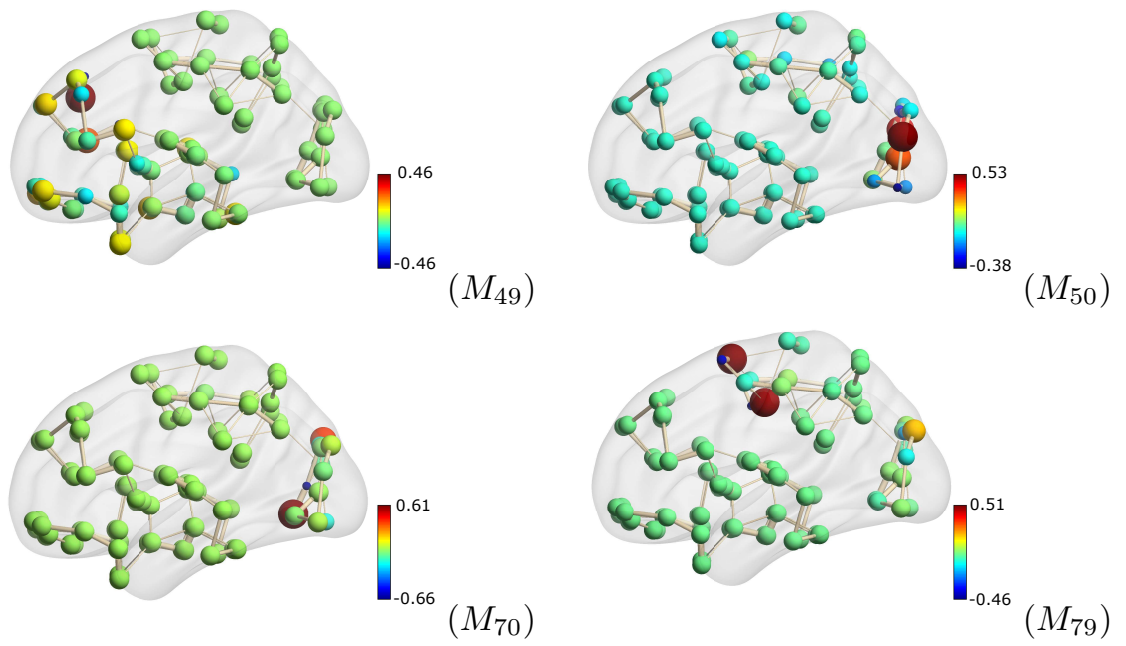

Figure 10: Significant frequency Modes $(M)$ in NT patients.
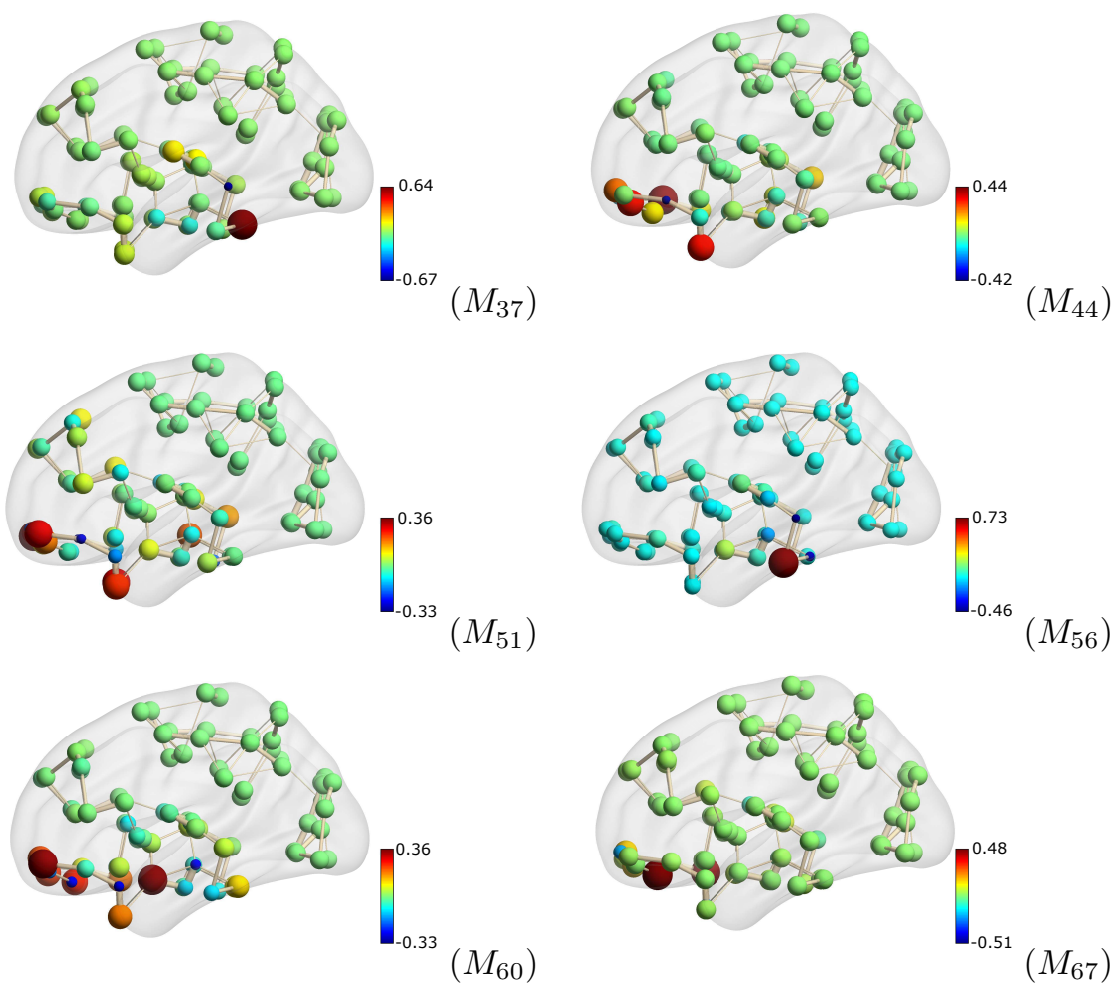

Figure 11: Significant frequency Modes $(M)$ in ASD. 


\subsection{Comparison with the literature}

The availability of the ABIDE dataset has surely made a major contribution in the increasing literature about data-driven ASD prediction, based on rsfMRI data. In this respect, the last few years have seen a significant interest for deep learning algorithms in attempt to predict ASD more accurately (Lu et al., 2020; Eslami \& Saeed, 2019; El-Gazzar et al., 2019; Heinsfeld et al., 2018). Recent works though tend to show that it may be possible to reach similar performances through less complex classification schemes such as linear SVMs (Thomas et al., 2020). The SVM classifier has also been commonly considered in several previous studies (Kazeminejad \& Sotero, 2019; Subbaraju et al., 2017). The linear version of the classifier is notably appreciated for its interpretability (Abraham et al., 2017).

The progress achieved on the ABIDE dataset is difficult to estimate from the available literature since the works differ in many respects, e.g. definition of inclusion criteria, segmentation in training and test sets, feature extraction/selection (Itani et al., 2019a). However, we can compare the present work with the literature from a general perspective and raise the following elements.

- Our framework yields favorable performances in comparison to the work of Abraham et al. (2017) which also aimed at achieving a form of interpretability, through the use of a linear SVM. This indicates the strength and pertinence of the approach that we propose for the extraction of discriminative features.

- The reported performances are getting close to $75 \%$ based on a decision tree, which is definitely promising while deep learning architectures and SVMs are the most common classifiers used in the literature related to the ABIDE dataset. Indeed, decision trees present a simple and readable structure in comparison to deep learning models. In comparison to SVMs, decision trees are directly focused on the most relevant training features, and provide explanations in the form of logical sequences, thus raising the interactions between the features. 
- The results show how the structural information may influence and improve the performance achieved by using only fMRI data. This interesting finding further develops the approach consisting of using functional connectivity, which has been commonly considered in the study of ASD and the ABIDE dataset so far (see e.g., Kazeminejad \& Sotero 2019; Dammu \& Bapi 2019; Heinsfeld et al. 2018; Abraham et al. 2017; Subbaraju et al. 2017).

\subsection{Limitations and future directions}

In terms of both performance and interpretation, the present study yields strong results given (i) the heterogeneity of the samples, and (ii) the simplistic definition of the brain topology. Indeed, our sample is heterogeneous by the range of the patients' ages (between 6 and 18 years old), the multisite nature of the data (seventeen sites contributed to the ABIDE-I dataset) and the range of profiles covered by the ASD spectrum. This disparity may incidentally explain the variability existing around the reported accuracies (Abraham et al., 2017). Studying larger and more homogeneous samples merits further investigations.

Moreover, the topological structure that we consider is only an approximation of the brain structural connectivity. The assessment of our approach against true topologies, i.e., deduced from individual DTI-based matrices, possibly thresholded to keep the most significant connections, is definitely interesting. The discriminative features would still be analyzed to raise influential frequency modes. They would however be unique to each person in terms of structural connectivity.

\section{Conclusion}

In this work, we extended the Graph Signal Processing (GSP) framework by introducing a new algorithm which classifies time-varying graph signals, with application to the identification of Autism Spectrum Disorder (ASD). The algorithm exploits the structure (i.e., brain topology) - function (i.e., fMRI-based 
activity) interplay of the brain to predict Autism Spectrum Disorder (ASD). In this context, we defined a brain graph as a set of regions of interest connected in terms of their topological distance. For such a purpose, we used the Graph Fourier Transform (GFT) which generalizes the notion of frequency analysis in an irregular domain. The resulting features were processed through an extension of the Fukunaga-Koontz transform to build discriminative markers for the classification of ASD and neurotypical patients. The approach was applied on the publicly available ABIDE dataset. We summarize below the main conclusions of this study.

- The proposed methodology performed favorably in comparison to stateof-the-art methods, on the basis of a decision tree as a predictive model.

- The analysis of the results reveals the influence of the frontal and temporal lobes in the diagnosis of the disorder. This finding is consistent with previous reports of the literature of neuroscience.

- In terms of brain activity, we show that the neuropathology may not be attributed to impairments in only low, medium and high frequency modes. Indeed, we observe the significant involvement of components that are picked in different parts of the frequency spectrum in the creation of the discriminative features.

These findings indicate that exploiting jointly structural and functional information of the brain regions is clearly a direction to be pursued in the study of ASD. We believe that our approach paves the way for a better understanding of the disease and thus the exploration of new research avenues by medical experts.

\section{Conflicts of interest}

None. 


\section{Acknowledgements}

Sarah Itani is a research fellow of the F.R.S.-FNRS. We thank Professors Philippe Fortemps, Arnaud Vandaele, Fabian Lecron (Faculty of Engineering, University of Mons), Professor Mandy Rossignol (Faculty of Psychology and Education, University of Mons, Belgium), Dr. Xiaowen Dong (University of Oxford), and Dr. Christine Choirat (Swiss Data Science Center - EPFL and ETH Zurich) for their advice and interest in this work.

\section{Appendix A. Appendices}

Appendix A.1. The pair-wise joint expectancy is semi-definite positive

We can prove that the pair-wise joint expectancy $\mathbf{S}_{i}$, as defined by Eq. 3, is positive semi-definite. For simplicity, we ignore the index $i$. By definition, a matrix $\mathbf{S}$ is positive semi-definite if:

$$
x^{T} \mathbf{S} x \geq 0 \quad \forall x \in \mathbb{R}^{n} .
$$

In this case, $\mathbf{S}=\mathbf{Y Y}^{T}$, which involves, $\forall x \in \mathbb{R}^{n}$ :

$$
x^{T} \mathbf{S} x=x^{T} \mathbf{Y} \mathbf{Y}^{T} x=\left(\mathbf{Y}^{T} x\right)^{T}\left(\mathbf{Y}^{T} x\right)=\left\|\mathbf{Y}^{T} x\right\|^{2} \geq 0 .
$$

Appendix A.2. The mean pair-wise joint expectancy is non invertible

Let us express the matrix $\mathbf{Y}_{i}$ of normalized GFT coefficients for a given patient $i$ as:

$$
\mathbf{Y}_{i}=\left(\hat{\mathbf{X}}_{i}-\mathbf{M}_{i}\right) \cdot \mathbf{R}_{i} \quad \text { with } \quad \mathbf{M}_{i}=\mathbf{U V}^{T} \mathbf{X}_{i}
$$

where $\mathbf{R}_{i}$ is a diagonal matrix including the norm of the columns of $\mathbf{Y}_{i}$ and $\mathbf{M}_{i}$ is a matrix including the mean of the columns of $\hat{\mathbf{X}}_{i}$; $\mathbf{U}$ is a $r \times r$ constant matrix including entries equal to $1 / r$.

Given Eq. A.1, an alternative expression for the mean joint expectancy matrix $\overline{\mathbf{S}}$ is:

$$
\overline{\mathbf{S}}=\frac{1}{n} \sum_{i}\left(\hat{\mathbf{X}}_{i}-\mathbf{M}_{i}\right) \cdot \mathbf{H}_{i} \cdot\left(\hat{\mathbf{X}}_{i}-\mathbf{M}_{i}\right)^{T}
$$


where, for the sake of simplicity, we denote $\mathbf{H}_{i}$ as:

$$
\frac{\mathbf{D}_{i}{ }^{2}}{\operatorname{Tr}\left(\mathbf{Y}_{i} \mathbf{Y}_{i}^{T}\right)}
$$

The matrix $\overline{\mathbf{S}}$ has a zero eigenvalue associated to a constant eigenvector, that is, if $q$ denotes a constant column vector:

$$
\overline{\mathbf{S}} q=0 .
$$

*** Proof

$$
\overline{\mathbf{S}} q=\frac{1}{n} \sum_{i}\left(\hat{\mathbf{X}}_{i}-\mathbf{M}_{i}\right) \cdot \mathbf{H}_{i} \cdot\left(\hat{\mathbf{X}}_{i}^{T}-\mathbf{M}_{i}^{T}\right) \cdot q
$$

Let us focus on the individual terms, i.e., for each $i$ :

$$
\begin{aligned}
\left(\hat{\mathbf{X}}_{\mathbf{i}}-\mathbf{M}_{i}\right) \cdot \mathbf{H}_{i} \cdot\left(\hat{\mathbf{X}}_{i}^{T}-\mathbf{M}_{i}^{T}\right) \cdot q \\
\quad=\hat{\mathbf{X}}_{i} \mathbf{H}_{i} \hat{\mathbf{X}}_{i}^{T} q-\hat{\mathbf{X}}_{i} \mathbf{H}_{i} \mathbf{M}_{i}^{T} q-\mathbf{M}_{i} \mathbf{H}_{i} \hat{\mathbf{X}}_{i}^{T} q+\mathbf{M}_{i} \mathbf{H}_{i} \mathbf{M}_{i}^{T} q .
\end{aligned}
$$

Yet $\hat{\mathbf{X}}_{i}^{T} q=\mathbf{M}_{i}^{T} q$. Indeed:

$$
\begin{aligned}
\mathbf{M}_{i}^{T} q & =\left(\mathbf{U V}^{T} \cdot \mathbf{X}_{i}\right)^{T} q \\
& =\left(\mathbf{V}^{T} \cdot \mathbf{X}_{i}\right)^{T} \mathbf{U}^{T} q \\
& =\hat{\mathbf{X}}_{i}^{T} \mathbf{U}^{T} q .
\end{aligned}
$$

As $\mathbf{U}$ is a square constant matrix, $\mathbf{U}^{T}=\mathbf{U}$. The vector $\mathbf{U} q$ includes the mean of each element of the vector $q$, which is a constant vector. Thus $\mathbf{U} q=q$ and

$$
\mathbf{M}_{i}^{T} q=\hat{\mathbf{X}}_{i}^{T} q
$$

which means that (A.3) sums to zero and thus, (A.2) also. This is related to the mean centering operation, executed over the columns of the matrices $\hat{\mathbf{X}}_{i}$. ${ }^{* * *}$

\section{Appendix A.3. The Newcomb's theorem}

For the sake of completeness, we recall here the main results of the theorem. 
Diagonalization (Newcomb, 1961). Let $\mathbf{A}$ and $\mathbf{B}$ be $n \times n$ real, symmetric, positive semi-definite matrices. Then there exists a real non-singular matrix $\mathbf{T}$ and real diagonal matrices $\mathbf{A}_{0}$ and $\mathbf{B}_{0}$ such that

$$
\begin{aligned}
& \mathbf{A}=\mathbf{T} \mathbf{A}_{0} \mathbf{T}^{T} \\
& \mathbf{B}=\mathbf{T} \mathbf{B}_{0} \mathbf{T}^{T}
\end{aligned}
$$

where

$$
\mathbf{A}_{0}=\operatorname{diagonal}\left[\mathbf{0}_{\mathbf{n}-\mathbf{a}}, \mathbf{I}_{\mathbf{a}}\right]
$$

if a denotes the rank of matrix $\mathbf{A}$.

Appendix A.4. Simultaneous diagonalization of the whitened matrices

Preliminaries. Let us show that $\overline{\mathbf{S}}^{\mathbf{A}^{\prime}}$ and $\overline{\mathbf{S}}^{\mathbf{N}^{\prime}}$ are positive semi-definite $(p s d)$ and have the following structures:

$$
\overline{\mathbf{S}}^{\mathbf{A}^{\prime}}=\left[\begin{array}{ccc}
0 & \ldots & 0 \\
\vdots & & \\
0 & \overline{\mathbf{S}}_{\mathbf{r}-\mathbf{1}}^{\mathbf{A}^{\prime}}
\end{array}\right] \quad \overline{\mathbf{S}}^{\mathbf{N}^{\prime}}=\left[\begin{array}{ccc}
0 & \ldots & 0 \\
\vdots & & \\
0 & \overline{\mathbf{S}}_{\mathbf{r}-\mathbf{1}}^{\mathbf{N}^{\prime}}
\end{array}\right] .
$$

We present here the reasoning for $\overline{\mathbf{S}}^{\mathbf{A}^{\prime}}$. The same applies to $\overline{\mathbf{S}}^{\mathbf{N}^{\prime}}$.

By definition, as the matrix of joint expectancy $\overline{\mathbf{S}}^{\mathbf{A}}$ is $p s d$, we have:

$$
x^{T} \overline{\mathbf{S}}^{\mathbf{A}} x \geq 0 \quad \forall x \in \mathbb{R}^{n} .
$$

We can show that $\overline{\mathbf{S}}^{\mathbf{A}^{\prime}}$ is $p s d$ as well. Indeed, let us set $x=\mathbf{Q}_{2}^{T} y, \forall y \in \mathbb{R}^{n}$. Then:

$$
\begin{aligned}
& y^{T} \overline{\mathbf{S}}^{\mathbf{A}^{\prime}} y=y^{T} \mathbf{Q}_{2} \overline{\mathbf{S}}^{A} \mathbf{Q}_{2}^{T} y=\left(\mathbf{Q}_{2}^{T} y\right)^{T} \overline{\mathbf{S}}^{\mathbf{A}}\left(\mathbf{Q}_{2}^{T} y\right) \\
& =x^{T} \overline{\mathbf{S}}^{\mathbf{A}} x \geq 0
\end{aligned}
$$

which involves $y^{T} \overline{\mathbf{S}}^{\mathbf{A}^{\prime}} y \geq 0 \Rightarrow \overline{\mathbf{S}}^{\mathbf{A}^{\prime}} \succeq 0$. As a $p s d$ matrix, the diagonal entries of $\overline{\mathbf{S}}^{\mathbf{A}^{\prime}}$ are positive (Golub \& Van Loan, 2012). Thus, to satisfy Eq. 7, the first main diagonal entry of $\overline{\mathbf{S}}^{\mathbf{A}^{\prime}}$ must be equal to zero; the corresponding row and column are zero, given the psd-ness of $\overline{\mathbf{S}}^{\mathbf{A}^{\prime}}$ (resp. $\overline{\mathbf{S}}^{\mathbf{N}^{\prime}}$ ) (Horn et al., 1990; Golub \& Van Loan, 2012). 
Diagonalization. In order to diagonalize $\overline{\mathbf{S}}^{\mathbf{A}^{\prime}}$, Newcomb (1961) proposes to diagonalize $\overline{\mathbf{S}}_{\mathbf{r}-\mathbf{1}}^{\mathbf{A}^{\prime}}$ by an orthogonal transformation $\mathbf{T}^{\prime}$ that we deduce through eigen-decomposition. The global transformation matrix $\mathbf{T}_{\mathbf{2}}$ constitutes of the following:

$$
\mathbf{T}_{\mathbf{2}}=\left[\begin{array}{c|ccc}
1 & 0 & \ldots & 0 \\
\hline 0 & & & \\
\vdots & & \mathbf{T}^{\prime} \\
0 & &
\end{array}\right]
$$

Thus, $\mathbf{T}_{\mathbf{2}}^{\mathbf{T}} \overline{\mathbf{S}}^{\mathbf{A}^{\prime}} \mathbf{T}_{\mathbf{2}}=\overline{\mathbf{S}}^{\mathbf{A}^{\prime \prime}}$, where $\overline{\mathbf{S}}^{\mathbf{A}^{\prime \prime}}$ is a diagonal matrix. Eq. 7 can be reformulated as:

$$
\begin{aligned}
& \mathbf{T}_{\mathbf{2}}^{\mathbf{T}} \text { diagonal }\left[\mathbf{0}, \mathbf{I}_{\mathbf{r}-\mathbf{1}}\right] \mathbf{T}_{\mathbf{2}}=\alpha_{\mathrm{A}} \mathbf{T}_{\mathbf{2}}^{\mathbf{T}} \overline{\mathbf{S}}^{\mathbf{A}^{\prime}} \mathbf{T}_{\mathbf{2}}+\alpha_{\mathrm{N}} \mathbf{T}_{\mathbf{2}}^{\mathbf{T}} \overline{\mathbf{S}}^{\mathbf{N}^{\prime}} \mathbf{T}_{\mathbf{2}} \\
& \Leftrightarrow \operatorname{diagonal}\left[\mathbf{0}, \mathbf{I}_{\mathbf{r}-\mathbf{1}}\right]=\alpha_{\mathrm{A}} \overline{\mathbf{S}}^{\mathbf{A}^{\prime \prime}}+\alpha_{\mathrm{N}} \overline{\mathbf{S}}^{\mathbf{N}^{\prime \prime}}
\end{aligned}
$$

Given that diagonal $\left[\mathbf{0}, \mathbf{I}_{\mathbf{r}-\mathbf{1}}\right]$ and $\overline{\mathbf{S}}^{\mathbf{A}^{\prime \prime}}$ are diagonal matrices, $\overline{\mathbf{S}}^{\mathbf{N}^{\prime \prime}}$ is diagonalizable, and it shares the same eigenvectors with $\overline{\mathbf{S}}^{\mathbf{A}^{\prime \prime}}$.

\section{Appendix A.5. Brain figures}

Table A.5 lists the ROIs of the AAL atlas grouped by partition, as suggested by Wang et al. (2012). These ROIs are labeled with the notations proposed by the BrainNet Software, which was used to draw the brain figures of the present paper. The ROI indexes related to the AAL atlas are also provided: odd (resp. even) numbers refer to ROIs from the left (resp. right) hemisphere. 


\begin{tabular}{|c|c|c|c|}
\hline Partition & INDEX L-R & ROI & LABEL \\
\hline \multirow{10}{*}{ Frontal } & 03-04 & Frontal_Sup & SFGdor \\
\hline & $05-06$ & Frontal_Sup_Orb & ORBsup \\
\hline & 07-08 & Frontal_Mid & MFG \\
\hline & $09-10$ & Frontal_Mid_Orb & ORBmid \\
\hline & $11-12$ & Frontal_Inf_Oper & IFGoperc \\
\hline & $13-14$ & Frontal_Inf_Tri & IFGtriang \\
\hline & $15-16$ & Frontal_Inf_Orb & ORBinf \\
\hline & $23-24$ & Frontal_Sup_Medial & SFGmed \\
\hline & $25-26$ & Frontal_Med_Orb & ORBsupmed \\
\hline & $27-28$ & Rectus & $\mathrm{REC}$ \\
\hline \multirow{9}{*}{ Parietal } & 01-02 & Precentral & PreCG \\
\hline & $19-20$ & Supp_Motor_Area & SMA \\
\hline & $57-58$ & Postcentral & PoCG \\
\hline & $59-60$ & Parietal_Sup & SPG \\
\hline & $61-62$ & Parietal_Inf & IPL \\
\hline & $63-64$ & SupraMarginal & SMG \\
\hline & $65-66$ & Angular & ANG \\
\hline & $67-68$ & Precuneus & PCUN \\
\hline & $69-70$ & Paracentral_Lobule & PCL \\
\hline \multirow{7}{*}{ Occipital } & $43-44$ & Calcarine & $\mathrm{CAL}$ \\
\hline & $45-46$ & Cuneus & CUN \\
\hline & $47-48$ & Lingual & LING \\
\hline & $49-50$ & Occipital_Sup & SOG \\
\hline & $51-52$ & Occipital_Mid & MOG \\
\hline & $53-54$ & Occipital_Inf & IOG \\
\hline & $55-56$ & Fusiform & FFG \\
\hline \multirow{11}{*}{ Temporal } & $17-18$ & Rolandic_Operr & ROL \\
\hline & $29-30$ & Insula & INS \\
\hline & $37-38$ & Hippocampus & HIP \\
\hline & $39-40$ & ParaHippocampal & PHG \\
\hline & $41-42$ & Amygdala & AMYG \\
\hline & $79-80$ & Heschl & HES \\
\hline & $81-82$ & Temporal_Sup & STG \\
\hline & $83-84$ & Temporal_Pole_Sup & TPOsup \\
\hline & $85-86$ & Temporal_Mid & MTG \\
\hline & $87-88$ & Temporal_Pole_Mid & TPOmid \\
\hline & $89-90$ & Temporal_Inf & ITG \\
\hline \multirow{3}{*}{ Cingulum } & $31-32$ & Cingulum_Ant & ACG \\
\hline & $33-34$ & Cingulum_Mid & DCG \\
\hline & $35-36$ & Cingulum_Post & PCG \\
\hline \multirow{5}{*}{ Subcortical } & $21-22$ & Olfactory & OLF \\
\hline & $71-72$ & Caudate & $\mathrm{CAU}$ \\
\hline & $73-74$ & Putamen & PUT \\
\hline & $75-76$ & Pallidum & PAL \\
\hline & $77-78$ & Thalamus & THA \\
\hline
\end{tabular}

Table A.5: AAL atlas (Xia et al., 2013; Wang et al., 2012; Tzourio-Mazoyer et al., 2002) 


\section{References}

Abhang, P. A., Gawali, B. W., \& Mehrotra, S. C. (2016). Introduction to EEGand Speech-Based Emotion Recognition. Academic Press.

Abraham, A., Milham, M. P., Di Martino, A., Craddock, R. C., Samaras, D., Thirion, B., \& Varoquaux, G. (2017). Deriving reproducible biomarkers from multi-site resting-state data: An autism-based example. NeuroImage, 147, $736-745$.

Alexander-Bloch, A. F., Vertes, P. E., Stidd, R., Lalonde, F., Clasen, L., Rapoport, J., Giedd, J., Bullmore, E. T., \& Gogtay, N. (2012). The anatomical distance of functional connections predicts brain network topology in health and schizophrenia. Cerebral cortex, 23, 127-138.

Autism Brain Imaging Data Exchange (2013). Welcome to the Autism Brain Imaging Data Exchange! http://fcon_1000.projects.nitrc.org/indi/ abide/. [Online; accessed 11-06-2020].

Autism Speaks (2020). DSM-5 Criteria. https://www.autismspeaks.org/ dsm-5-criteria. [Online; accessed 11-06-2020].

Baars, B. J., \& Gage, N. M. (2010). Cognition, brain, and consciousness: Introduction to cognitive neuroscience. Academic Press.

Bullmore, E., \& Sporns, O. (2009). Complex brain networks: graph theoretical analysis of structural and functional systems. Nature Reviews Neuroscience, 10, 186-198.

Chen, H., Duan, X., Liu, F., Lu, F., Ma, X., Zhang, Y., Uddin, L. Q., \& Chen, H. (2016). Multivariate classification of autism spectrum disorder using frequency-specific resting-state functional connectivitya multi-center study. Progress in Neuro-Psychopharmacology and Biological Psychiatry, 64, 1-9.

Craddock, C., Benhajali, Y., Chu, C., Chouinard, F., Evans, A., Jakab, A., Khundrakpam, B. S., Lewis, J. D., Li, Q., Milham, M. et al. (2013). The neuro 
bureau preprocessing initiative: open sharing of preprocessed neuroimaging data and derivatives. Neuroinformatics, 41 .

Dammu, P. S., \& Bapi, R. S. (2019). Employing temporal properties of brain activity for classifying autism using machine learning. In International Conference on Pattern Recognition and Machine Intelligence (pp. 193-200). Springer.

Di Martino, A., Yan, C.-G., Li, Q., Denio, E., Castellanos, F. X., Alaerts, K., Anderson, J. S., Assaf, M., Bookheimer, S. Y., Dapretto, M. et al. (2014). The autism brain imaging data exchange: Towards large-scale evaluation of the intrinsic brain architecture in autism. Molecular psychiatry, 19, 659 .

Doshi-Velez, F., \& Kim, B. (2017). Towards a rigorous science of interpretable machine learning. arXiv:1702.08608v2.

El-Gazzar, A., Quaak, M., Cerliani, L., Bloem, P., van Wingen, G., \& Thomas, R. M. (2019). A Hybrid 3DCNN and 3DC-LSTM Based Model for 4D SpatioTemporal fMRI Data: An ABIDE Autism Classification Study. In OR 2.0 Context-Aware Operating Theaters and Machine Learning in Clinical Neuroimaging (pp. 95-102). Springer.

Eslami, T., \& Saeed, F. (2019). Auto-ASD-network: A technique based on deep learning and support vector machines for diagnosing autism spectrum disorder using fMRI data. In Proceedings of the 10th ACM International Conference on Bioinformatics, Computational Biology and Health Informatics (pp. 646$651)$.

Frank, E., Hall, M. A., \& Witten, I. H. (2016). The WEKA Workbench. Online Appendix for "Data Mining: Practical Machine Learning Tools and Techniques". Morgan Kaufmann.

Fukunaga, K. (2013). Introduction to statistical pattern recognition. Elsevier. 
Fukunaga, K., \& Koontz, W. L. (1970). Application of the karhunen-loeve expansion to feature selection and ordering. IEEE Transactions on computers, $100,311-318$.

Goch, C. J., Oztan, B., Stieltjes, B., Henze, R., Hering, J., Poustka, L., Meinzer, H.-P., Yener, B., \& Maier-Hein, K. H. (2014). Global changes in the connectome in autism spectrum disorders. In Computational Diffusion MRI and Brain Connectivity (pp. 239-247). Springer.

Goldberg, M., Mena, I., \& Miller, B. (1999). Frontal and temporal lobe dysfunction in autism and other related disorders: ADHD and OCD. Latin American Journal of Nuclear Medicine, .

Golub, G. H., \& Van Loan, C. F. (2012). Matrix computations volume 3. JHU Press.

Heinsfeld, A. S., Franco, A. R., Craddock, R. C., Buchweitz, A., \& Meneguzzi, F. (2018). Identification of autism spectrum disorder using deep learning and the ABIDE dataset. NeuroImage: Clinical, 17, 16-23.

Hirata, K., Egashira, K., Harada, K., Nakashima, M., Hirotsu, M., Isomura, S., Watanuki, T., Matsubara, T., Kaku, Y., Kaneyuki, H. et al. (2018). Differences in frontotemporal dysfunction during social and non-social cognition tasks between patients with autism spectrum disorder and schizophrenia. Scientific reports, 8, 3014.

Horn, R. A., Horn, R. A., \& Johnson, C. R. (1990). Matrix analysis. Cambridge university press.

Huang, W., Goldsberry, L., Wymbs, N. F., Grafton, S. T., Bassett, D. S., \& Ribeiro, A. (2016). Graph frequency analysis of brain signals. J. Sel. Topics Signal Processing, 10, 1189-1203.

Huo, X. (2004). A statistical analysis of fukunaga-koontz transform. IEEE Signal Processing Letters, 11, 123-126. 
Itani, S., Lecron, F., \& Fortemps, P. (2019a). Data Mining for ADHD \& ASD prediction based on resting-state fMRI signals: A literature review. In 31st Benelux Conference on Artificial Intelligence (BNAIC 2019) and the 28th Belgian Dutch Conference on Machine Learning (Benelearn 2019). CEURWS.org, online CEUR-WS.org/Vol-2491/paper20.pdf.

Itani, S., Lecron, F., \& Fortemps, P. (2019b). Specifics of medical data mining for diagnosis aid: A survey. Expert Systems with Applications, 118, 300-314.

Kana, R. K., Uddin, L. Q., Kenet, T., Chugani, D., \& Müller, R.-A. (2014). Brain connectivity in autism. Frontiers in Human Neuroscience, 8, 349.

Kassraian-Fard, P., Matthis, C., Balsters, J. H., Maathuis, M. H., \& Wenderoth, N. (2016). Promises, pitfalls, and basic guidelines for applying machine learning classifiers to psychiatric imaging data, with autism as an example. Frontiers in psychiatry, 7, 177.

Kazeminejad, A., \& Sotero, R. C. (2019). Topological properties of resting-state fmri functional networks improve machine learning-based autism classification. Frontiers in neuroscience, 12, 1018.

Ktena, S. I., Parisot, S., Ferrante, E., Rajchl, M., Lee, M., Glocker, B., \& Rueckert, D. (2018). Metric learning with spectral graph convolutions on brain connectivity networks. NeuroImage, 169, 431-442.

Lauvin, M.-A., Martineau, J., Destrieux, C., Andersson, F., Bonnet-Brilhault, F., Gomot, M., El-Hage, W., \& Cottier, J.-P. (2012). Functional morphological imaging of autism spectrum disorders: current position and theories proposed. Diagnostic and interventional imaging, 93, 139-147.

Lu, H., Liu, S., Wei, H., \& Tu, J. (2020). Multi-kernel fuzzy clustering based on auto-encoder for fMRI functional network. Expert Systems with Applications, (p. 113513). 
Maximo, J. O., Cadena, E. J., \& Kana, R. K. (2014). The implications of brain connectivity in the neuropsychology of autism. Neuropsychology review, 24, $16-31$.

Medaglia, J. D., Huang, W., Karuza, E. A., Kelkar, A., Thompson-Schill, S. L., Ribeiro, A., \& Bassett, D. S. (2018). Functional alignment with anatomical networks is associated with cognitive flexibility. Nature Human Behaviour, 2, 156 .

Ménoret, M., Farrugia, N., Pasdeloup, B., \& Gripon, V. (2017). Evaluating graph signal processing for neuroimaging through classification and dimensionality reduction. In Signal and Information Processing (GlobalSIP), 2017 IEEE Global Conference on (pp. 618-622). IEEE.

Mueller, S., Keeser, D., Samson, A. C., Kirsch, V., Blautzik, J., Grothe, M., Erat, O., Hegenloh, M., Coates, U., Reiser, M. F. et al. (2013). Convergent findings of altered functional and structural brain connectivity in individuals with high functioning autism: a multimodal MRI study. PloS one, 8, e67329.

Nair, A., Treiber, J. M., Shukla, D. K., Shih, P., \& Müller, R.-A. (2013). Impaired thalamocortical connectivity in autism spectrum disorder: A study of functional and anatomical connectivity. Brain, 136, 1942-1955.

Newcomb, R. W. (1961). On the simultaneous diagonalization of two semidefinite matrices. Quarterly of Applied Mathematics, 19, 144-146.

Ortega, A., Frossard, P., Kovačević, J., Moura, J. M., \& Vandergheynst, P. (2018). Graph signal processing: Overview, challenges, and applications. Proceedings of the IEEE, 106, 808-828.

Poustka, L., Jennen-Steinmetz, C., Henze, R., Vomstein, K., Haffner, J., \& Sieltjes, B. (2012). Fronto-temporal disconnectivity and symptom severity in children with autism spectrum disorder. The World Journal of Biological Psychiatry, 13, 269-280. 
Preprocessed Connectomes Project (2014). ABIDE Preprocessed. http: //preprocessed-connectomes-project.org/abide/. [Online; accessed 1106-2020].

Rane, P., Cochran, D., Hodge, S. M., Haselgrove, C., Kennedy, D., \& Frazier, J. A. (2015). Connectivity in autism: A review of MRI connectivity studies. Harvard review of psychiatry, 23, 223.

Ray, S., Miller, M., Karalunas, S., Robertson, C., Grayson, D. S., Cary, R. P., Hawkey, E., Painter, J. G., Kriz, D., Fombonne, E. et al. (2014). Structural and functional connectivity of the human brain in autism spectrum disorders and attention-deficit/hyperactivity disorder: A rich club-organization study. Human brain mapping, 35, 6032-6048.

Shuman, D. I., Narang, S. K., Frossard, P., Ortega, A., \& Vandergheynst, P. (2013). The emerging field of signal processing on graphs: Extending highdimensional data analysis to networks and other irregular domains. IEEE Signal Processing Magazine, 30, 83-98.

Subbaraju, V., Suresh, M. B., Sundaram, S., \& Narasimhan, S. (2017). Identifying differences in brain activities and an accurate detection of autism spectrum disorder using resting state functional-magnetic resonance imaging: A spatial filtering approach. Medical image analysis, 35, 375-389.

Thomas, R. M., Gallo, S., Cerliani, L., Zhutovsky, P., El-Gazzar, A., \& van Wingen, G. (2020). Classifying autism spectrum disorder using the temporal statistics of resting-state functional mri data with 3D convolutional neural networks. Frontiers in Psychiatry, 11, 440.

Tolan, E., \& Isik, Z. (2018). Graph theory based classification of brain connectivity network for autism spectrum disorder. In International Conference on Bioinformatics and Biomedical Engineering (pp. 520-530). Springer.

Tzourio-Mazoyer, N., Landeau, B., Papathanassiou, D., Crivello, F., Etard, O., Delcroix, N., Mazoyer, B., \& Joliot, M. (2002). Automated anatomical 
labeling of activations in SPM using a macroscopic anatomical parcellation of the MNI MRI single-subject brain. NeuroImage, 15, 273-289.

Uddin, L. Q., Supekar, K., \& Menon, V. (2013). Reconceptualizing functional brain connectivity in autism from a developmental perspective. Frontiers in human neuroscience, 7, 458.

Vargason, T., Grivas, G., Hollowood-Jones, K. L., \& Hahn, J. (2020). Towards a multivariate biomarker-based diagnosis of autism spectrum disorder: Review and discussion of recent advancements. In Seminars in Pediatric Neurology (p. 100803). Elsevier.

Vissers, M. E., Cohen, M. X., \& Geurts, H. M. (2012). Brain connectivity and high functioning autism: A promising path of research that needs refined models, methodological convergence, and stronger behavioral links. Neuroscience \&3 Biobehavioral Reviews, 36, 604-625.

Wang, J., Calhoun, V. D., Stephen, J. M., Wilson, T. W., \& Wang, Y.-p. (2018). Integration of network topological features and graph Fourier transform for fMRI data analysis. In Biomedical Imaging (ISBI 2018), 2018 IEEE 15th International Symposium on (pp. 92-96). IEEE.

Wang, Z., Liu, J., Zhong, N., Qin, Y., Zhou, H., \& Li, K. (2012). Changes in the brain intrinsic organization in both on-task state and post-task resting state. Neuroimage, 62, 394-407.

Xia, M., Wang, J., \& He, Y. (2013). BrainNet Viewer: a network visualization tool for human brain connectomics. PloS one, 8, e68910. 